ISSN 1855-3966 (printed edn.), ISSN 1855-3974 (electronic edn.)

ARS MATHEMATICA CONTEMPORANEA 19 (2020) 51-60

https://doi.org/10.26493/1855-3974.2034.dad

(Also available at http://amc-journal.eu)

\title{
Association schemes with a certain type of $p$-subschemes*
}

\author{
Wasim Abbas ${ }^{\dagger}$ (D), Mitsugu Hirasaka \\ Department of Mathematics, College of Sciences, Pusan National University, \\ 63 Beon-gil 2, Busandaehag-ro, Geumjung-gu, Busan 609-735, Korea
}

Received 4 July 2019, accepted 16 July 2020, published online 2 November 2020

\begin{abstract}
In this article, we focus on association schemes with some properties derived from the orbitals of a transitive permutation group $G$ with a one-point stabilizer $H$ satisfying $H<N_{G}(H)<N_{G}\left(N_{G}(H)\right) \unlhd G$ and $\left|N_{G}\left(N_{G}(H)\right)\right|=p^{3}$ where $p$ is a prime. By a corollary of our main result we obtain some inequality which corresponds to the fact $\left|G: N_{G}\left(N_{G}(H)\right)\right| \leq p+1$.
\end{abstract}

Keywords: Association schemes, p-schemes.

Math. Subj. Class. (2020): 05E15, 05E30

\section{Introduction}

Let $G$ be a finite group with a subgroup $H$ which satisfies

$$
H<N_{G}(H)<N_{G}\left(N_{G}(H)\right) \unlhd G \quad \text { and } \quad\left|N_{G}\left(N_{G}(H)\right)\right|=p^{3}
$$

where $p$ is a prime. In this article we focus on association schemes axiom-zing some properties derived from the orbitals of the action of $G$ on $G / H$.

We shall recall some terminologies to show that the definition of coherent configurations is derived from properties of the binary relations obtained from a permutation group. Let $G$ be a permutation group of a finite set $\Omega$. Then $G$ acts on $\Omega \times \Omega$ by its entry-wise action, i.e.,

$$
(\alpha, \beta)^{x}:=\left(\alpha^{x}, \beta^{x}\right) \text { for } \alpha, \beta \in \Omega \text { and } x \in G .
$$

We denote the set of orbits of the action of $G$ on $\Omega \times \Omega$ by $\operatorname{Inv}(G)$, which satisfies the following conditions:

\footnotetext{
* This work was supported by BK21 Dynamic Math Center, Department of Mathematics at Pusan National University.

${ }^{\dagger}$ Corresponding author.

E-mail addresses: wasimabbas@ pusan.ac.kr (Wasim Abbas), hirasaka@ pusan.ac.kr (Mitsugu Hirasaka)
} 
(i) The diagonal relation $1_{\Omega}$ is a union of elements of $\operatorname{Inv}(G)$;

(ii) For each $s \in \operatorname{Inv}(G)$ we have $s^{*} \in \operatorname{Inv}(G)$ where $s^{*}:=\{(\alpha, \beta) \mid(\beta, \alpha) \in s\}$;

(iii) For all $s, t, u \in \operatorname{Inv}(G)$ we have $\sigma_{s} \sigma_{t}=\sum_{u \in S} c_{s t}^{u} \sigma_{u}$ for $c_{s t}^{u} \in \mathbb{N}$ uniquely determined by $s, t, u$ where $\sigma_{u}$ is the adjacency matrix of $u$, i.e., $\left(\sigma_{u}\right)_{\alpha, \beta}=1$ if $(\alpha, \beta) \in u$ and $\left(\sigma_{u}\right)_{\alpha, \beta}=0$ if $(\alpha, \beta) \notin u$.

A coherent configuration is a pair $(\Omega, S)$ of a finite set $\Omega$ and a partition $S$ of $\Omega \times \Omega$ which satisfies the conditions obtained from the above by replacing $\operatorname{Inv}(G)$ by $S$. We say that a coherent configuration $(\Omega, S)$ is schurian if $S=\operatorname{Inv}(G)$ for some permutation group $G$ of $\Omega$, and it is homogeneous or an association scheme if $1_{\Omega} \in S$ (see [2] and [3] for its background).

Suppose that $G$ has a subgroup $H$ which satisfies (1.1). Then $|H|=p,\left|N_{G}(H)\right|=p^{2}$ and for each $g \in G$ we have the following:

(i) $|H g H| /|H| \in\{1, p\}$ and $\left|N_{G}(H) g N_{G}(H)\right| /\left|N_{G}(H)\right| \in\{1, p\}$;

(ii) $|H g H| /|H|=1$ if and only if $g \in N_{G}(H)$;

(iii) $\left|N_{G}(H) g N_{G}(H)\right| /\left|N_{G}(H)\right|=1$ if and only if $g \in N_{G}\left(N_{G}(H)\right)$;

(iv) $N_{G}\left(N_{G}(H)\right)$ is the smallest normal subgroup of $G$ containing $H$.

Since $G$ acts faithfully and transitively on the set of right cosets of $H$ in $G$ by its right multiplication, it induces a schurian association scheme $(\Omega, S)$ where $\Omega=\{H x \mid x \in G\}$ and $S=\operatorname{Inv}(G)$ such that, for each $s \in S$ we have the following:

(i) $n_{s} \in\{1, p\}$ where $n_{s}:=c_{s s^{*}}^{1_{\Omega}}$;

(ii) $\mathbf{O}_{\theta}(S)$ forms a group of order $p$ where $\mathbf{O}_{\theta}(S):=\left\{s \in S \mid n_{s}=1\right\}$;

(iii) $\mathbf{O}^{\theta}(S)=\left\{s \in S \mid s s^{*} s=s\right\}$ where $\mathbf{O}^{\theta}(S)$ is the thin residue of $S$ (see Section 2, [9] or [10] for its definition).

The following is our main result:

Theorem 1.1. Let $(\Omega, S)$ be an association scheme with $\mathbf{O}_{\theta}(S)<\mathbf{O}^{\theta}(S)$ such that $n_{s} \in$ $\{1, p\}$ for each $s \in S$ and $n_{\mathbf{O}^{\theta}(S)}=p^{2}$ where $p$ is a prime. Then $|\Omega| \leq p^{2}(p+1)$.

In [4] they give a criterion on association schemes whose thin residue $\mathbf{O}^{\theta}(S)$ induces the subschemes isomorphic to either

$$
C_{p^{2}}, \quad C_{p} \times C_{p} \quad \text { or } \quad C_{p} \prec C_{p}
$$

Here we denote $(G, \operatorname{Inv}(G))$ by $G$ when $G$ acts on itself by its right multiplication and we denote the wreath product of one scheme $(\Delta, U)$ by another scheme $(\Gamma, V)$ by $(\Delta, U)$ ? $(\Gamma, V)$, i.e.,

$$
(\Delta, U) \curlywedge(\Gamma, V):=\left(\Delta \times \Gamma,\left\{1_{\Gamma} \otimes u \mid u \in U\right\} \cup\left\{v \otimes U \mid v \in V \backslash\left\{1_{\Gamma}\right\}\right\}\right)
$$

where

$$
\begin{aligned}
1_{\Gamma} \otimes u & :=\left\{\left(\left(\delta_{1}, \gamma\right),\left(\delta_{2}, \gamma\right)\right) \mid\left(\delta_{1}, \delta_{2}\right) \in u, \gamma \in \Gamma\right\} \quad \text { and } \\
v \otimes U & :=\left\{\left(\left(\delta_{1}, \gamma_{1}\right),\left(\delta_{2}, \gamma_{2}\right)\right) \mid \delta_{1}, \delta_{2} \in \Delta,\left(\gamma_{1}, \gamma_{2}\right) \in v\right\}
\end{aligned}
$$


For the case of $\mathbf{O}^{\theta}(S) \simeq C_{p^{2}}$ we can apply the main result in [7] to conclude that $(\Omega, S)$ is schurian. For the case of $\mathbf{O}^{\theta}(S) \simeq C_{p} \times C_{p}$ we can say that $|\Omega| \leq p^{2}\left(p^{2}+p+1\right)$ under the assumption that $n_{s}=p$ for each $s \in S \backslash \mathbf{O}^{\theta}(S)$. For the case of $\mathbf{O}^{\theta}(S) \simeq C_{p} \prec C_{p}$ we had no progression for the last five years.

In [6] all association schemes of degree 27 are classified by computational enumeration, and there are three pairs of non-isomorphic association schemes with $\mathbf{O}^{\theta}(S) \simeq C_{3}$ ? $C_{3}$ which are algebraic isomorphic. These examples had given an impression that we need some complicated combinatorial argument to enumerate $p$-schemes $(\Omega, S)$ with $\mathbf{O}^{\theta}(S) \simeq$ $C_{p}\left\{C_{p}\right.$ and $\left\{n_{s} \mid s \in S \backslash \mathbf{O}^{\theta}(S)\right\}=\{p\}$. The following reduces our argument to the $p$-schemes of degree $p^{3}$ where an association scheme $(\Omega, S)$ is called a $p$-scheme if $|s|$ is a power of $p$ for each $s \in S$ :

Corollary 1.2. For each $p$-scheme $(\Omega, S)$ with $\mathbf{O}^{\theta}(S) \simeq C_{p} \prec C_{p}$, if $n_{s}=p$ for each $s \in S \backslash \mathbf{O}^{\theta}(S)$, then $|\Omega|=p^{3}$.

In the proof of Theorem 1.1 the theory of coherent configurations plays an important role through the thin residue extension which is a way of construction of coherent configurations from association schemes (see [5, Theorem 2.1] or [8]). The following is the kernel of our paper:

Theorem 1.3. For each coherent configuration $(\Omega, S)$ whose fibers are isomorphic to $C_{p} 2$ $C_{p}$, if $|s|=p^{3}$ for each $s \in S$ with $\sigma_{s} \sigma_{s}=0$, then either $|\Omega| \leq p^{2}(p+1)$ or $s s^{*} s=s$ for each $s \in S$.

In Section 2 we prepare necessary terminologies on coherent configurations. In Section 3 we prove our main results.

\section{Preliminaries}

Throughout this section, we assume that $(\Omega, S)$ is a coherent configuration. An element of $\Omega$ and an element of $S$ are called a point and a basis relation, respectively. Furthermore, $|\Omega|$ and $|S|$ are called the degree and rank of $(\Omega, S)$, respectively. For all $\alpha, \beta \in \Omega$ the unique element in $S$ containing $(\alpha, \beta)$ is denoted by $r(\alpha, \beta)$. For $s \in S$ and $\alpha \in \Omega$ we set

$$
\alpha s:=\{\beta \in \Omega \mid(\alpha, \beta) \in s\} .
$$

A subset $\Delta$ of $\Omega$ is called a fiber of $(\Omega, S)$ if $1_{\Delta} \in S$. For each $s \in S$, there exists a unique pair $(\Delta, \Gamma)$ of fibers such that $s \subseteq \Delta \times \Gamma$. For fibers $\Delta, \Gamma$ of $(\Omega, S)$ we denote the set of $s \in S$ with $s \subseteq \Delta \times \Gamma$ by $S_{\Delta, \Gamma}$, and we set $S_{\Delta}:=S_{\Delta, \Delta}$. It is easily verified that $\left(\Delta, S_{\Delta}\right)$ is a homogeneous coherent configuration. Now we define the complex product on the power set of $S$ as follows: For all subsets $T$ and $U$ of $S$ we set

$$
T U:=\left\{s \in S \mid c_{t u}^{s}>0 \text { for some } t \in T \text { and } u \in U\right\}
$$

where the singleton $\{t\}$ in the complex product is written without its parenthesis.

The following equations are frequently used without any mention:

Lemma 2.1. Let $(\Omega, S)$ be a coherent configuration. Then we have the following:

(i) For all $r, s \in S$, if $r s \neq \emptyset$, then $n_{r} n_{s}=\sum_{t \in S} c_{r s}^{t} n_{t}$;

(ii) For all $r, s, t \in S$ we have $|t| c_{r s}^{t^{*}}=|r| c_{s t}^{r^{*}}=|s| c_{t r}^{s^{*}}$; 
(iii) For all $r, s \in S$ we have $|\{t \in S \mid t \in r s\}| \leq \operatorname{gcd}\left(n_{r}, n_{s}\right)$.

For $T \subseteq S_{\Delta, \Gamma}$ we set

$$
n_{T}:=\sum_{t \in T} n_{t}
$$

Here we mention closed subsets, their subschemes and factor scheme according to the terminologies given in [10]. Let $(\Omega, S)$ be an association scheme and $T \subseteq S$. We say that a non-empty subset $T$ of $S$ is closed if $T T^{*} \subseteq T$ where

$$
T^{*}:=\left\{t^{*} \mid t \in T\right\}
$$

equivalently $\bigcup_{t \in T} t$ is an equivalence relation on $\Omega$ whose equivalence classes are

$$
\{\alpha T \mid \alpha \in \Omega\}
$$

where $\alpha T:=\{\beta \in \Omega \mid(\alpha, \beta) \in t$ for some $t \in T\}$. Let $T$ be a closed subset of $S$ and $\alpha \in \Omega$. It is well-known (see [9]) that

$$
(\Omega, S)_{\alpha T}:=(\alpha T,\{t \cap(\alpha T \times \alpha T) \mid t \in T\})
$$

is an association scheme, called the subscheme of $(\Omega, S)$ induced by $\alpha T$, and that

$$
(\Omega, S)^{T}:=(\Omega / T, S / / T)
$$

is also an association scheme where

$$
\begin{aligned}
\Omega / T & :=\{\alpha T \mid \alpha \in \Omega\}, \quad S / / T=\left\{s^{T} \mid s \in S\right\} \quad \text { and } \\
s^{T} & :=\{(\alpha T, \beta T) \mid(\gamma, \delta) \in s \text { for some }(\gamma, \delta) \in \alpha T \times \beta T\},
\end{aligned}
$$

which is called the factor scheme of $(\Omega, S)$ over $T$.

We say that a closed subset $T$ is thin if $n_{t}=1$ for each $t \in T$, and $\mathbf{O}_{\theta}(S)$ is called the thin radical of $S$, and the smallest closed subset $T$ such that $S / / T$ is thin is called the thin residue of $S$, which is denoted by $\mathbf{O}^{\theta}(S)$.

\section{Proof of the main theorem}

Let $(\Omega, S)$ be a coherent configuration whose distinct fibers are $\Omega_{1}, \Omega_{2}, \ldots, \Omega_{m}$. For all integers $i, j$ with $1 \leq i, j \leq m$ we set

$$
S_{i j}:=S_{\Omega_{i}, \Omega_{j}} \quad \text { and } \quad S_{i}:=S_{i i}
$$

Throughout this section we assume that $\left(\Omega_{i}, S_{i}\right) \simeq C_{p} \prec C_{p}$ for $i=1,2, \ldots, m$ where $p$ is a prime and $C_{p} \prec C_{p}$ is a unique non-thin $p$-scheme of degree $p^{2}$ up to isomorphism.

For $s \in S$ we say that $s$ is regular if $s s^{*} s=\{s\}$ and we denote by $R$ the set of regular elements in $S$.

Lemma 3.1. For each regular element $s \in S_{i j}$ with $n_{s}=p$ we have

$$
\sigma_{s} \sigma_{s^{*}}=p\left(\sum_{t \in \mathbf{O}_{\theta}\left(S_{i}\right)} \sigma_{t}\right) \quad \text { and } \quad \sigma_{s^{*}} \sigma_{s}=p\left(\sum_{t \in \mathbf{O}_{\theta}\left(S_{j}\right)} \sigma_{t}\right) \text {. }
$$

In particular, $s s^{*}=\mathbf{O}_{\theta}\left(S_{i}\right)$ and $s^{*} s=\mathbf{O}_{\theta}\left(S_{j}\right)$. 
Proof. Notice that $\left\{1_{\Omega_{i}}\right\} \subsetneq s s^{*} \subset S_{i}$ and $t s=\{s\}$ for each $t \in s s^{*}$. Since $\left\{t \in S_{i} \mid\right.$ $t s=\{s\}\}$ is a closed subset of valency at most $n_{s}$, it follows from $\left(\Omega_{i}, S_{i}\right) \simeq C_{p} \prec C_{p}$ that $s s^{*}=\mathbf{O}_{\theta}\left(S_{i}\right)$, and hence for each $t \in s s^{*}$

$$
c_{s s^{*}}^{t}=c_{s t}^{s} n_{s^{*}} / n_{t^{*}}=p .
$$

This implies that $\sigma_{s} \sigma_{s^{*}}=p\left(\sum_{t \in \mathbf{O}_{\theta}\left(S_{i}\right)} \sigma_{t}\right)$. By the symmetric argument we have $\sigma_{s^{*}} \sigma_{s}=$ $p\left(\sum_{t \in \mathbf{O}_{\theta}\left(S_{j}\right)} \sigma_{t}\right)$.

Lemma 3.2. For each non-regular element $s \in S_{i j}$ with $n_{s}=p$ we have

$$
\sigma_{s} \sigma_{s^{*}}=p \sigma_{1_{\Omega_{i}}}+\sum_{u \in S_{i} \backslash \mathbf{O}_{\theta}\left(S_{i}\right)} \sigma_{u} \quad \text { and } \quad \sigma_{s^{*}} \sigma_{s}=p \sigma_{1_{\Omega_{j}}}+\sum_{u \in S_{j} \backslash \mathbf{O}_{\theta}\left(S_{j}\right)} \sigma_{u} .
$$

Proof. Notice that $\left\{t \in S_{i} \mid t s=\{s\}\right\}=\left\{1_{\Omega_{i}}\right\}$, otherwise, $s$ is regular or $n_{s}=p^{2}$, a contradiction. This implies that the singletons $t s$ with $t \in \mathbf{O}_{\theta}\left(S_{i}\right)$ are distinct elements of valency $p$. Since

$$
p^{2}=\left|\Omega_{j}\right|=\sum_{s \in S_{i j}} n_{s} \geq \sum_{t \in \mathbf{O}_{\theta}\left(S_{i}\right)} n_{t s}=p+p+\cdots+p=p^{2},
$$

it follows that $\mathbf{O}_{\theta}\left(S_{i}\right) s=S_{i j}$.

We claim that $S_{i} \backslash \mathbf{O}_{\theta}\left(S_{i}\right) \subseteq s s^{*}$. Let $u \in S_{i} \backslash \mathbf{O}_{\theta}\left(S_{i}\right)$. Then there exists $t \in \mathbf{O}_{\theta}\left(S_{i}\right)$ such that $u \in t s s^{*}$ since $u \in S_{i j} s^{*}=\mathbf{O}_{\theta}\left(S_{i}\right) s s^{*}$. This implies that $u=t^{*} u \subseteq t^{*}\left(t s s^{*}\right)=$ $s s^{*}$.

By the claim with $p^{2}=n_{s} n_{s^{*}}=\sum_{t \in S_{i}} c_{s s^{*} t} n_{t}$ and $c_{s s^{*} 1_{\Omega_{i}}}=n_{s}=p$ we have the first statement, and the second statement is obtained by the symmetric argument.

For the remainder of this section we assume that $n_{s}=p$ for each $s \in \bigcup_{i \neq j} S_{i j}$.

Lemma 3.3. The set $\bigcup_{s \in R} s$ is an equivalence relation on $\Omega$.

Proof. Since $1_{\Omega_{i}} \in S_{i} \subseteq R$ for $i=1,2, \ldots, m, \bigcup_{s \in R} s$ is reflexive. Since $s s^{*} s=\{s\}$ is equivalent to $s^{*} s s^{*}=\left\{s^{*}\right\}, \bigcup_{s \in R} s$ is symmetric.

Let $\alpha \in \Omega_{i}, \beta \in \Omega_{j}$ and $\gamma \in \Omega_{k}$ with $r(\alpha, \beta), r(\beta, \gamma) \in R$. Then we have

$$
r(\alpha, \gamma) r(\alpha, \gamma)^{*} \subseteq r(\alpha, \beta) r(\beta, \gamma) r(\beta, \gamma)^{*} r(\alpha, \beta)^{*}
$$

If one of $r(\alpha, \beta), r(\beta, \gamma)$ is thin, then $(\alpha, \gamma) r(\alpha, \gamma)^{*}$, and hence $r(\alpha, \gamma) \in R$. Now we assume that both of them are non-thin. Since $r(\beta, \gamma) r(\beta, \gamma)^{*}=\mathbf{O}_{\theta}\left(S_{j}\right)=r(\alpha, \beta)^{*} r(\alpha, \beta)$, it follows that

$$
r(\alpha, \gamma) r(\alpha, \gamma)^{*} \subseteq r(\alpha, \beta) r(\alpha, \beta)^{*}=\mathbf{O}_{\theta}\left(S_{i}\right)
$$

Applying Lemma 3.1 and 3.2 we obtain that $r(\alpha, \gamma)$ is regular, and hence $\bigcup_{s \in R} s$ is transitive.

Lemma 3.4. The set $\bigcup_{s \in N} s$ is an equivalence relation on $\Omega$ where $N:=\bigcup_{i=1}^{m} S_{i} \cup$ $(S \backslash R)$. 
Proof. Since $1_{\Omega_{i}} \in S_{i} \subseteq N$ for $i=1,2, \ldots, m, \bigcup_{s \in N} s$ is reflexive. By Lemma 3.3, $\bigcup_{s \in R}$ is symmetric, so that $\bigcup_{s \in N} s$ is symmetric.

Let $\alpha \in \Omega_{i}, \beta \in \Omega_{j}$ and $\gamma \in \Omega_{k}$ with $r(\alpha, \beta), r(\beta, \gamma) \in N$. Since $\bigcup_{i=1}^{m} S_{i} \subseteq R$, it follows from Lemma 3.3 that it suffices to show that

$$
r(\alpha, \gamma) \in S \backslash R
$$

under the assumption that

$$
r(\alpha, \beta), r(\beta, \gamma) \in S \backslash R \quad \text { with } i \neq k .
$$

Suppose the contrary, i.e., $r(\alpha, \gamma) \in R$. Then, by Lemma 3.3, $S_{i k} \subseteq R$. Since

$$
r(\alpha, \beta) r(\beta, \gamma) \subseteq S_{i k} \subseteq R
$$

it follows that

$$
\mathbf{O}_{\theta}\left(S_{i}\right) r(\alpha, \beta) r(\beta, \gamma)=r(\alpha, \beta) r(\beta, \gamma) .
$$

On the other hand, we have

$$
\mathbf{O}_{\theta}\left(S_{i}\right) r(\alpha, \beta) r(\beta, \gamma)=S_{i j} r(\beta, \gamma)=S_{i k} .
$$

Thus, $r(\alpha, \beta) r(\beta, \gamma)=S_{i k}$. Since $i \neq k$, each element of $S_{i k}$ has valency $p$, and hence,

$$
\sigma_{s_{1}} \sigma_{s_{2}}=\sum_{u \in S_{i k}} \sigma_{u}
$$

where $s_{1}:=r(\alpha, \beta)$ and $s_{2}:=r(\beta, \gamma)$. By Lemma 3.2,

$$
p^{2}=\left\langle\sigma_{s_{1}} \sigma_{s_{2}}, \sigma_{s_{1}} \sigma_{s_{2}}\right\rangle=\left\langle\sigma_{s_{1}}^{*} \sigma_{s_{1}}, \sigma_{s_{2}} \sigma_{s_{2}}^{*}\right\rangle=p^{2}+p(p-1),
$$

a contradiction where $\langle$,$\rangle is the inner product defined by$

$$
\langle A, B\rangle:=1 / p^{2} \operatorname{tr}\left(A B^{*}\right) \quad \text { for all } A, B \in M_{\Omega}(\mathbb{C}) .
$$

Therefore, $\bigcup_{s \in N} s$ is transitive.

Lemma 3.5. We have either $R=S$ or $N=S$.

Proof. Suppose $R \neq S$. Let $\alpha, \beta \in \Omega$ with $r(\alpha, \beta) \in R$. Since $R \neq S$, there exists $\gamma \in \Omega$ with $r(\alpha, \gamma) \in N$. Notice that $r(\beta, \gamma) \in R \cup N$. By Lemma 3.3, $r(\beta, \gamma) \in N$, and hence, by Lemma 3.4,

$$
r(\alpha, \beta) \in R \cap N=\bigcup_{i=1}^{m} S_{i} .
$$

Since $\alpha, \beta \in \Omega$ are arbitrarily taken, it follows that

$$
R=\bigcup_{i=1}^{m} S_{i} \text { and } N=S .
$$

Lemma 3.6. Suppose that $S=N$ and $s_{1} \in S_{i j}, s_{2} \in S_{j k}$ and $s_{3} \in S_{i k}$ with distinct $i, j, k$. Then $\sigma_{s_{1}} \sigma_{s_{2}}=\sigma_{s_{3}}\left(\sum_{t \in \mathbf{O}_{\theta}\left(S_{k}\right)} a_{t} \sigma_{t}\right)$ for some non-negative integers $a_{t}$ with $\sum_{t \in \mathbf{O}_{\theta}\left(S_{k}\right)} a_{t}=p, \sum_{t \in \mathbf{O}_{\theta}\left(S_{k}\right)} a_{t}^{2}=2 p-1$ and for each $u \in \mathbf{O}_{\theta}\left(S_{k}\right) \backslash\left\{1_{\Omega_{k}}\right\}$, $\sum_{t \in \mathbf{O}_{\theta}\left(S_{k}\right)} a_{t} a_{t u}=p-1$. 
Proof. Since $s_{1} s_{2} \subseteq S_{i j}=s_{3} \mathbf{O}_{\theta}\left(S_{k}\right), \sigma_{s_{1}} \sigma_{s_{2}}=\sum_{t \in \mathbf{O}_{\theta}\left(S_{k}\right)} a_{t} \sigma_{s_{3} t}$ for some nonnegative integers $a_{t}$. Since $\sigma_{s_{3} t}=\sigma_{s_{3}} \sigma_{t}$ and

$$
p^{2}=n_{s_{1}} n_{s_{2}}=\sum_{t \in \mathbf{O}_{\theta}\left(S_{k}\right)} a_{t} n_{s_{3} t}=p \sum_{t \mathbf{O}_{\theta}\left(S_{j}\right)} a_{t},
$$

it remains to show the last two equalities on $a_{t}$ with $t \in \mathbf{O}_{\theta}\left(S_{j}\right)$. Expanding $\sigma_{s_{2}}^{*} \sigma_{s_{1}}^{*} \sigma_{s_{1}} \sigma_{s_{2}}$ by two ways we obtain from Lemma 3.2 that

$$
\begin{aligned}
\left(2 p^{2}-p\right) \sigma_{1_{\Omega_{j}}}+\left(p^{2}-p\right) \sum_{t \in \mathbf{O}_{\theta}\left(S_{j}\right) \backslash\left\{1_{\Omega_{j}}\right\}} \sigma_{t} & +\left(p^{2}-2 p\right) \sum_{u \in S_{j} \backslash \mathbf{O}_{\theta}\left(S_{j}\right)} \sigma_{u} \\
& =\sum_{t \in \mathbf{O}_{\theta}\left(S_{k}\right)} a_{t} \sigma_{t}^{*} \sigma_{s_{3}}^{*} \sigma_{s_{3}} \sum_{t \in \mathbf{O}_{\theta}\left(S_{k}\right)} a_{t} \sigma_{t} .
\end{aligned}
$$

Therefore, we conclude from Lemma 3.2 that

$$
p \sum_{t \in \mathbf{O}_{\theta}\left(S_{k}\right)} a_{t}^{2}=2 p^{2}-p \quad \text { and } \quad p \sum_{t \in \mathbf{O}_{\theta}\left(S_{k}\right)} a_{t} a_{t u}=p^{2}-p
$$

for each $u \in \mathbf{O}_{\theta}\left(S_{k}\right)$ with $u \neq 1_{\Omega_{k}}$.

For the remainder of this section we assume that

$$
S=N
$$

For $i=1,2, \ldots, m$ we take $\alpha_{i} \in \Omega_{i}$ and we define $t_{i} \in S_{i}$ such that $t_{1} \in \mathbf{O}_{\theta}\left(S_{1}\right) \backslash\left\{1_{\Omega_{1}}\right\}$, and for $i=2,3, \ldots, m, t_{i}$ is a unique element in $\mathbf{O}_{\theta}\left(S_{i}\right)$ with $r\left(\alpha_{1} t_{1}, \alpha_{i} t_{i}\right)=r\left(\alpha_{1}, \alpha_{i}\right)$. Then $C_{p}$ acts semi-regularly on $\Omega$ such that

$$
\Omega \times C_{p} \rightarrow \Omega, \quad\left(\beta_{i}, t^{j}\right) \mapsto \beta_{i} t_{i}^{j},
$$

where $C_{p}=\langle t\rangle$ and $\beta_{i}$ is an arbitrary element in $\Omega_{i}$.

Lemma 3.7. The above action acts semi-regularly on $\Omega$ as an automorphism of $(\Omega, S)$.

Proof. Since $C_{p}$ acts regularly on each of geometric coset of $\mathbf{O}_{\theta}\left(S_{i}\right)$ for $i=1,2, \ldots, m$, the action is semi-regular on $\Omega$. By the definition of $\left\{t_{i}\right\}$, it is straightforward to show that $r\left(\alpha_{1}, \alpha_{i}\right)$ is fixed by the action on $\Omega \times \Omega$, and hence each element of $\bigcup_{j=2}^{m} S_{1 j} \cup$ $S_{j 1}$ is also fixed since $S_{1 j}=\mathbf{O}_{\theta}\left(S_{1}\right) r\left(\alpha_{1}, \alpha_{j}\right)$. Let $s \in S_{i j}$ with $2 \leq i, j$. Notice that $r\left(\alpha_{i}, \alpha_{1}\right) r\left(\alpha_{1}, \alpha_{j}\right)$ is a proper subset of $S_{i j}$ by Lemma 3.6. This implies that $s$ is obtained as the intersection of some of $t_{i}^{k} r\left(\alpha_{i}, \alpha_{1}\right) r\left(\alpha_{1}, \alpha_{j}\right)$ with $0 \leq k \leq p-1$, and hence $s$ is fixed.

For each $i=1,2, \ldots, m$ we take $\left\{\alpha_{i k} \mid k=1,2, \ldots, m\right\}$ to be a complete set of representatives with respect to the equivalence relation $\bigcup_{t \in \mathbf{O}_{\theta}\left(S_{i}\right)} t$ on $\Omega_{i}$.

Lemma 3.8. For each $s \in S_{i j}$ with $i \neq j$ and all $k, l=1,2, \ldots, p$ there exists a unique $h(s)_{k l} \in \mathbb{Z}_{p}$ such that $r\left(\alpha_{i k}, \alpha_{j l} t^{h(s)_{k l}}\right)=s$. Moreover, if $s_{1} \in S_{i j}$ and $t^{a} \in \mathbf{O}_{\theta}\left(S_{k}\right)$ with $s_{1}=s^{a}$, then $h\left(s_{1}\right)_{k l}=h(s)_{k l}+$ a for all $k, l=1,2, \ldots, m$. 
Proof. Since $\mathbf{O}_{\theta}\left(S_{j}\right)$ acts regularly on $S_{i j}$ by its right multiplication, the first statement holds. The second statement is obtained by a direct computation.

Lemma 3.9. For each $s \in S_{i j}$ with $i \neq j$ and all $k, l=1,2, \ldots, p$ we have

$$
s \cap\left(\alpha_{i k} \mathbf{O}_{\theta}\left(S_{i}\right) \times \alpha_{j l} \mathbf{O}_{\theta}\left(S_{j}\right)\right)=\left\{\left(\alpha_{i k} t_{i}^{a}, \alpha_{j l} t_{j}^{b}\right) \mid b-a=h(s)_{k l}\right\} .
$$

Proof. Notice that

$$
r\left(\alpha_{i k} t_{i}^{a}, \alpha_{j l} t_{j}^{b}\right)=\left(t_{i}^{a}\right)^{*} r\left(\alpha_{i k}, \alpha_{j l}\right) t_{j}^{b}=r\left(\alpha_{i k}, \alpha_{j l}\right) t_{j}^{b-a} .
$$

Since $r\left(\alpha_{i k}, \alpha_{j l} t^{h(s)_{k l}}\right)=s$ by Lemma 3.8, it follows that $r\left(\alpha_{i k} t_{i}^{a}, \alpha_{j l} t_{j}^{b}\right)=s$ if and only if $b-a=h(s)_{k l}$.

Proposition 3.10. For each $s \in S_{i j}$ with $i \neq j$ the matrix $\left(h(s)_{k l}\right) \in M_{p \times p}\left(\mathbb{Z}_{p}\right)$ satisfies that, for all distinct $k_{1}, k_{2} \in\{1,2, \ldots, p\}$,

$$
\left\{h(s)_{k_{1}, l}-h(s)_{k_{2}, l} \mid l=1,2, \ldots, p\right\}=\mathbb{Z}_{p} .
$$

In other word the matrix is a generalized Hadamard matrix of degree $p$ over $\mathbb{Z}_{p}$, equivalently, the matrix $\left(\xi^{\left.h(s)_{k l}\right)} \in M_{p \times p}(\mathbb{C})\right.$ is a complex Hadamard matrix of Butson type $(p, p)$ where $\xi$ is a primitive p-th root of unity.

Proof. Notice that, for all distinct $k, l$, by Lemma 3.9,

$$
\left\{\gamma \in \Omega \mid r\left(\alpha_{i k} t_{i}^{a}, \gamma\right)=r\left(\alpha_{i l} t_{i}^{b}, \gamma\right)=s\right\}
$$

equals

$$
\bigcup_{r=1}^{p}\left\{\alpha_{j r} t_{j}^{c} \mid c-a=h(s)_{k r}, c-b=h(s)_{l r}\right\} .
$$

Since the upper one is a singleton by Lemma 3.2, there exists a unique $r \in\{1,2, \ldots, p\}$ such that $b-a=h(s)_{k r}-h(s)_{l r}$. Since $a$ and $b$ are arbitrarily taken, the first statement holds.

The second statement holds since $\sum_{i=0}^{p-1} x^{i}$ is the minimal polynomial of $\xi$ over $\mathbb{Q}$.

We shall write the matrix $\left(\xi^{h(s)_{k l}}\right)$ as $H(s)$. For $s \in S_{i j}$ with $i \neq j$, the restriction of $\sigma_{s}$ to $\Omega_{i} \times \Omega_{j}$ can be viewed as a $(p \times p)$-matrix whose $(k, l)$-entry is the matrix $P_{i}^{h(s)_{k l}}$ where $P_{i}$ is the permutation matrix corresponding to the mapping $\beta_{i} \mapsto \beta_{i} t_{i}$ where we may assume that $P_{i}=P_{j}$, say $P$, for all $i, j=1,2, \ldots, m$ by Lemma 3.7. Notice that $H(s)$ is obtained from $\left(P^{h(s)_{k l}}\right)$ by sending $P^{h(s)_{k l}}$ to $\xi^{h(s)_{k l}}$.

Proposition 3.11. For all $s_{1} \in S_{i j}, s_{2} \in S_{j k}$ and $s_{3} \in S_{i k}$ with distinct $i, j, k$ we have $H\left(s_{1}\right) H\left(s_{2}\right)=\alpha H\left(s_{3}\right)$ for some $\alpha \in \mathbb{C}$ with $|\alpha|=\sqrt{p}$.

Proof. By Lemma 3.6, $H\left(s_{1}\right) H\left(s_{2}\right)=H\left(s_{3}\right)\left(\sum_{i=0}^{p-1} a_{i} \xi^{i}\right)$ for some $a_{i} \in \mathbb{Z}$ where $a_{i}=$ $c_{t_{k}^{i}}$. Thus, it suffices to show that $\left|\left(\sum_{i=0}^{p-1} a_{i} \xi^{i}\right)\right|^{2}=p$. By Lemma 3.6, the left hand side equals

$$
\sum_{i=0}^{p-1} \sum_{j=0}^{p-1} a_{i} a_{j} \xi^{i-j}=\sum_{i=0}^{p-1} a_{i}^{2}+\sum_{i=1}^{p-1} \sum_{j=0}^{p-1} a_{j} a_{i+j} \xi^{i}=(2 p-1)+(p-1)(-1)=p .
$$


Corollary 3.12. Let $s_{i}:=r\left(\alpha_{1}, \alpha_{i}\right)$ for $i=2,3, \ldots, m$ and $\mathbf{B}_{i}$ denote the basis consisting of the rows of $H\left(s_{i}\right), i=2,3, \ldots, m$, and $\mathbf{B}_{1}$ be the standard basis. Then $\left\{\mathbf{B}_{1}, \mathbf{B}_{2}, \ldots\right.$, $\left.\mathbf{B}_{m}\right\}$ is a mutually unbiased bases for $\mathbb{C}^{p}$, and $m \leq p+1$.

Proof. The first statement is an immediate consequence of Proposition 3.10, and the second statement follows from a well-known fact that the number of mutually unbiased bases for $\mathbb{C}^{n}$ is at most $n+1$ (see [1]).

Proof of Theorem 1.3. Suppose that $R \neq S$. Then $N=S$ and the theorem follows from Corollary 3.12 .

Proof of Theorem 1.1. Since $n_{\mathbf{O}^{\theta}(S)}=p^{2}$ and $\mathbf{O}_{\theta}(S)<\mathbf{O}^{\theta}(S)$, it follows from [5, Theorem 2.1] (or see [8]) that the thin residue extension of $(\Omega, S)$ is a coherent configuration with all fibers isomorphic to $C_{p}<C_{p}$ such that each basic relation out of the fibers has valency $p$.

We claim that $S=N$. Otherwise, $S=R$, which implies that $\left\langle s s^{*} \mid s \in S\right\rangle$ has valency $p$. Since $\mathbf{O}^{\theta}(S)=\left\langle s s^{*} \mid s \in S\right\rangle$ (see [9]), it contradicts that $\mathbf{O}^{\theta}(S)$ has valency $p^{2}$.

By the claim, $S=N$. Since the number of fibers of the thin residue extension of $(\Omega, S)$ equals $\left|\Omega / \mathbf{O}^{\theta}(S)\right|$, the theorem follows from Theorem 1.3.

Proof of Corollary 1.2. Since $(\Omega, S)$ is a $p$-scheme and $\mathbf{O}^{\theta}(S) \simeq C_{p} \times C_{p},|\Omega|$ is a power of $p$ greater than $p^{2}$. By Theorem 1.1, $|\Omega| \leq(p+1) p^{2}$, and hence, $|\Omega|=p^{3}$.

\section{ORCID iDs}

Wasim Abbas (D) https://orcid.org/0000-0002-1706-1462

\section{References}

[1] S. Bandyopadhyay, P. O. Boykin, V. Roychowdhury and F. Vatan, A new proof for the existence of mutually unbiased bases, Algorithmica 34 (2002), 512-528, doi:10.1007/ s00453-002-0980-7.

[2] E. Bannai and T. Ito, Algebraic Combinatorics I: Association Schemes, The Benjamin/Cummings Publishing, Menlo Park, CA, 1984.

[3] A. E. Brouwer, A. M. Cohen and A. Neumaier, Distance-Regular Graphs, volume 18 of Ergebnisse der Mathematik und ihrer Grenzgebiete, Springer-Verlag, Berlin, 1989, doi: 10.1007/978-3-642-74341-2.

[4] J. R. Cho, M. Hirasaka and K. Kim, On $p$-schemes of order $p^{3}$, J. Algebra 369 (2012), 369-380, doi:10.1016/j.jalgebra.2012.06.026.

[5] S. A. Evdokimov and I. N. Ponomarenko, Schemes of relations of the finite projective plane, and their extensions, Algebra i Analiz 21 (2009), 90-132, http://mi.mathnet.ru/ aa 996 , St. Petersburg Math. J. 21 (2010), 65-93, doi:10.1090/s1061-0022-09-01086-3.

[6] A. Hanaki and I. Miyamoto, Classification of association schemes of small order, Discrete Math. 264 (2003), 75-80, doi:10.1016/s0012-365x(02)00551-4.

[7] M. Hirasaka and P.-H. Zieschang, Sufficient conditions for a scheme to originate from a group, J. Comb. Theory Ser. A 104 (2003), 17-27, doi:10.1016/s0097-3165(03)00104-3.

[8] M. Muzychuk and I. Ponomarenko, On quasi-thin association schemes, J. Algebra 351 (2012), 467-489, doi:10.1016/j.jalgebra.2011.11.012. 
[9] P.-H. Zieschang, An Algebraic Approach to Association Schemes, volume 1628 of Lecture Notes in Mathematics, Springer-Verlag, Berlin, 1996, doi:10.1007/bfb0097032.

[10] P.-H. Zieschang, Theory of Association Schemes, Springer Monographs in Mathematics, Springer-Verlag, Berlin, 2005, doi:10.1007/3-540-30593-9. 